Proc. of Eighth International Conference On Advances in Civil, Structural and Mechanical Engineering - ACSM 2018

Copyright (C) Institute of Research Engineers and Doctors, USA. All rights reserved.

ISBN: 978-1-63248-154-2 doi: 10.15224/978-1-63248-154-2-10

\title{
Discharge Modeling in Smooth and Rough Compound Channels Using Genetic Programming
}

\section{[Alok Adhikari ${ }^{1}, \mathrm{~N}$ Adhikari, ${ }^{2}$ and K C Patra ${ }^{3}$ ]}

\begin{abstract}
Discharge results observed from the experimental channels for smooth and rough surfaces, along with data from a compound river channel are used in the Genetic Programming. Model equations are derived for prediction of discharge in compound channel for various types of channel surfaces. Five hydraulic parameters are used for developing the model equations. Models derived are tested and compared with other soft computing techniques. Evaluations of all the approaches are carried out using five performance parameters. Finally, the effect of parameters responsible for the flow behavior is shown through sensitivity analysis. GP is found to give the most promising results. This work aims to benefit the researchers engaged in modeling of discharge using machine learning techniques.
\end{abstract}

Keywords - cnn, fuzzy, ces, anfis, gp

\section{Introduction}

In hydrology a reliable assessment for discharge of a compound river section encompassing a deep main along with floodplains are subject of extensive research. The quantum of flow is very complex. The prediction of same helps in overall design, operation and maintenance of streams and its periphery. The overall conveyance of the compound channels is a function of stage and various other parameters like the hydraulics radius, cross sectional area, wetted perimeter, bed slope, roughness, shear, depth ratio, width ratio, sinuosity, viscosity, gravitational acceleration, momentum transfer mechanism etc. The detail literature study reveals that very limited works have been made where laboratory data collected in controlled environment are used for prediction. Mohanty et al. [1] used laboratory data for developing flow equations and tried to use them to compare with the flow behavior of river Batu [2] using a conventional method. Though the soft computing models like Artificial Neural Network (ANN) and Adaptive Neuro Fuzzy Inference System (ANFIS) are used for prediction of discharge but derivation of a model equation is very complex. During prediction, ANN modeling reduces the tedious effort of experimentation and complexity in computation [ $3 \& 4$ ]. In case there is shortage of data due to any error or physical phenomena then synthetic data can be reconstructed [5]. The models with artificial intelligence do not explicitly follow physical principles inside a system [6]. They constitute a universal approximation of the input and output and have the capability to reconstruct missing data. While among ANN modelling approaches Back Propagation Network (BPN) and CASCADE equations are established for input parameter (Stage) and output (Discharge), but are more complex [7 \& 8]. In ANFIS the presence of multiple parameters as inputs, makes it difficult to derive model equations [9].

To study and analyze the flow behavior, experimental channel is set up. Laboratory experimental data of Flood Channel Facility, Wallingford England are available globally [10-13] and are included in the data set. Apart from experimental data, the actual river data is used to build the data set. The current work analyses river data along with the laboratory data to get a generalized solution to the above problems. Impacts of the hydraulic parameters to the flow behavior need to be studied. To

Research Scholar, Department of Civil Engineering, NIT Rourke,India,

${ }^{2}$ Biju Patnaik University of Technology, Rourkela, India and

${ }^{3}$ Professor, Department of Civil Engineering, NIT Rourkela, India. overcome this, attempts have been made to study and apply the Genetic Programming (GP) [14-16]. Due to a forced restriction on GP tree depth, the evolved mathematical models are compact. Hence the influence of input parameters is easy to study.

\section{Experimental Arrangement}

Experiments are conducted in a straight compound channel with symmetrical flood plains in a flume of dimensions measuring $12 \mathrm{~m} \times 2 \mathrm{~m} \times 0.6 \mathrm{~m}$ in the Hydraulics Engineering laboratory of the National Institute of Technology Rourkela, India. Fabrication of channel is made with Perspex sheet of 0.006 meter having a uniform Manning`s $n$ value of 0.01 . The compound channel has the width ratio $(\alpha)$ as 15.75 and the aspect ratio $(\delta)$ of trapezoidal main channel is 1.5. the details of experimental channel shown in Fig.1 can be found in our earlier work [9]. The experimental channel section is shown in Fig.2 and the photographs for the entire set up are shown in Fig. 3(a-h).

In order to study the effect of roughness on the flow behavior during high floods experiments were carried out in different runs. First one was with plane surface and next three runs were carried out with different roughening materials to provide the effect of vegetation [9].

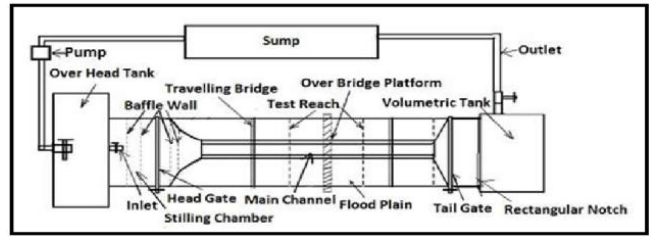

Figure 1. Schematic drawing of whole experimental tilting flume

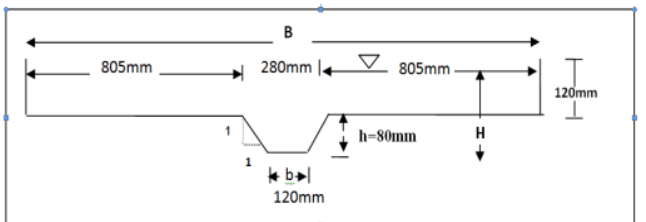

Figure 2. Straight Compound Channel Section

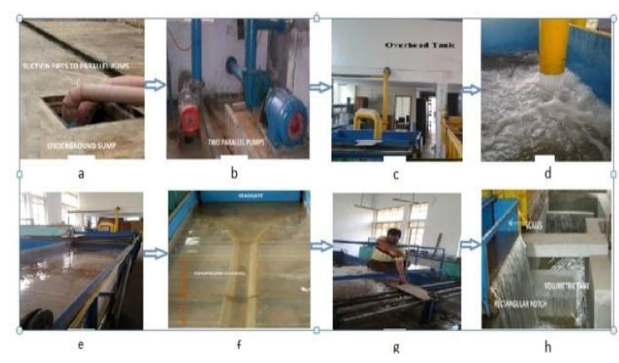

Figure 3. Photographs of whole experimental system

\section{Data Collection}

In the current work, present experimental set up data is combined with other researcher's data [10-13]. Then actual river data is also collected from two gauging stations 
namely Panposh and Gomlai in Sundergarh district of Odisha state for River Bramhani. Data sets observed from these sets of experimental channels ( smooth channel = data set $\mathrm{I}$, rough channel $=$ data set II, smooth + rough $=$ data set III, river data $=$ data set IV and combination of all above mentioned data sets taken as data set V) are grouped and named as they are used in the analysis. Five different channel parameters that are affecting stage discharge relations are selected for the analysis [1]. These parameters are: (1) Width ratio $\alpha$, (2) roughness coefficient of flood plain $n_{f p}$, (3) bed slope $S_{0}$, (4) relative depth of flood plane to total depth $\beta$ and (5) hydraulic radius $R_{T}$.

\section{Iv. Artificial Intelligence Techniques}

Artificial Intelligence (AI) techniques are the best alternative to classical techniques to model environmental systems. AI techniques can be case-based reasoning, rulebased systems, ANNs, Fuzzy Inference models, genetic algorithms, cellular automata, multi-agent systems, swarm intelligence and hybrid systems etc. ANN is a very vigorous technique to develop immense relationship between input and output variables. Using various types of ANN such as Back Propagation Network, Cascade, Radial Basis Function (RBF), and Elman Neural Network, discharge predictions are carried out for the corresponding stages and reported in our previous works [7 \& 8]. Similarly Fuzzy logic developed by Zadeh is used successfully for prediction of discharge. ANFIS is a very popular technique and is used by many researchers [17 \& 18]. The same has been used by our earlier reported work [9]. In the current study, emphasis is given to another robust tool, Genetic Programming- a multi gene symbolic regression technique.

\section{v. Genetic Programming}

Genetic programming (GP) is an optimization tool motivated from general biology. It randomly generates a population of computer programs for optimization. Here the computer programs are internally represented as tree structures. Then mutation and crossover is carried out on best performing trees to find a new population. This process is iterated until the population contains the programs that solve the task well. For the current research, the open source MATLAB toolbox called GPTIPS is used as it is developed for specific purpose of performing symbolic regression. It employs a unique type of symbolic regression called multigene symbolic regression [16] that evolves linear combinations of non linear transformations of the input variables. In standard symbolic regression techniques, the evolved models are not restricted to low orders depending on the supplied data sets. Whereas in GPTIPS the transformation is restricted to be low order (by restricting the GP tree depth) irrespective of the predictor response (input-output) data sets even when there are a large set of input parameters. A brief overview of the multigene low order GP approach is given in the next section. GP model is composed of nodes, which resembles a tree structure and thus, it is known as GP tree. Nodes are

the elements either from a functional set or terminal set. A functional set may include arithmetic operators $(+,-, *, /)$, mathematical functions $(\sin (),. \cos ($.$) , tanh or \ln ()$.$) ,$
Boolean operators (AND, OR, NOT, etc), logical expressions (IF, or THEN) or any other suitable functions defined by the users, whereas the terminal set include variables (like $x_{1}, x_{2}, x_{3}$, etc) or constants (like 1,2,3,4 etc.) or both. The functions and terminals are randomly chosen to form a GP tree with a root node and the branches extending from each function node to end in terminal nodes as shown in Fig. 4 for the expression $y=a x_{1}+\log$ $\mathrm{bx}_{2}$, $\mathrm{a}$ and $\mathrm{b}$ being the coefficients.

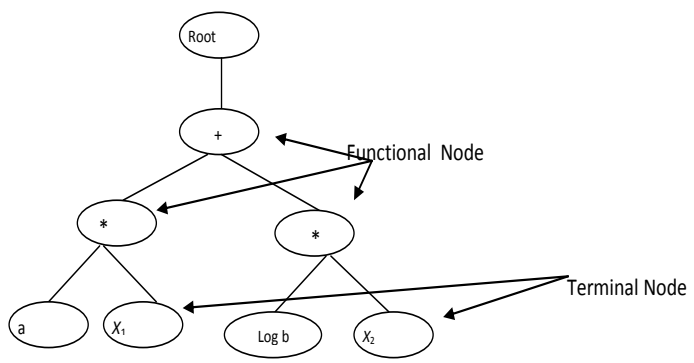

Figure 4. GP Tree for expression $\mathrm{ax}_{1}+\log \mathrm{b} \mathrm{x}_{2}$

\section{A. Discharge Prediction Using GPTIPS}

From previous studies it is observed that different hydraulic parameters play crucial role for discharge carrying capacity in a channel. Using artificial neural networks with stage as single input to predict discharge as the output, different network models are derived. It is observed the model equations derived are very complex. Therefore hydraulic characteristics like bed slope, hydraulic radius, roughness coefficient, aspect ratio, relative depth of compound channel are taken as input for predicting discharge. The generalized model equation is represented as:

$\mathrm{Q}=\sum_{i=1}^{n} F\left(X, f(X), b_{i}\right)+b_{0}$

where $\mathrm{Q}$ stands for Discharge, $\mathrm{F}$ is the function created by GP model, $\mathrm{X}$ is the vector of input variables $\left\{\alpha, n_{f p}, S_{0}\right.$, $\beta$ and $\left.R_{T}\right\}, b_{i}$ represents constants, $\mathrm{f}$ is user defined function, $\mathrm{n}$ is the number of terms of target expression and $\mathrm{b}_{0}$ is bias.

\section{B. GPTIPS Run Settings}

A GPTIPS run settings is performed for all the above data sets. Initially a set of GP trees, as per the data sets of each model are randomly generated using various functions and terminals assigned. The fitness criteria are calculated by the objective function, which determines the quality of each individual competing with the rest of the population. At each generation, a new population is created by implementing various evolutionary mechanisms like reproduction, crossover and mutation of the selected GP trees entering into the mating pool. The new population then replaces the existing population. This process is iterated until the termination criterion, which can be either a threshold fitness value or maximum number of generations is satisfied. The best GP model, based on its fitness value is selected as the result of genetic programming. The default GPTIPS multigene symbolic regression function is used in order to minimize the root mean squared prediction error on the testing data. Table 1 describes the best GP model derived for each data set with population size, number of generations, tournament size, depth of tree and maximum number of genes. 
Proc. of Eighth International Conference On Advances in Civil, Structural and Mechanical Engineering - ACSM 2018 Copyright $@$ Institute of Research Engineers and Doctors, USA. All rights reserved.

ISBN: 978-1-63248-154-2 doi: 10.15224/978-1-63248-154-2-10

TABLE I. DESCRIPTION OF GP MODELS FOR DIFFERENT DATA

\begin{tabular}{|c|c|c|c|c|c|c|c|}
\hline $\begin{array}{c}\text { Data } \\
\text { Set }\end{array}$ & \begin{tabular}{|c|} 
Training \\
data \\
(Initial \\
population)
\end{tabular} & $\begin{array}{l}\text { Testing } \\
\text { data }\end{array}$ & $\begin{array}{c}\text { No. of } \\
\text { Generations }\end{array}$ & $\begin{array}{l}\text { Tourna- } \\
\text { Ment } \\
\text { size }\end{array}$ & $\mathrm{D}_{\text {max }}$ & $\mathrm{G}_{\text {max }}$ & $\begin{array}{c}\text { Function } \\
\text { Node } \\
\text { set }\end{array}$ \\
\hline 1 & 120 & 20 & 77 & 4 & 3 & 5 & \multirow{5}{*}{\begin{tabular}{|l}
,+ \\
,- \\
$*$, \\
tanh, \\
plog. \\
square
\end{tabular}} \\
\hline II & 28 & 10 & 0 & 4 & 4 & 5 & \\
\hline III & 150 & 28 & 12 & 4 & 3 & 5 & \\
\hline IV & 50 & 24 & 48 & 4 & 4 & 5 & \\
\hline $\mathrm{v}$ & 222 & 50 & 206 & 4 & 4 & 5 & \\
\hline
\end{tabular}

\section{Interpretation of Results}

In the GP modeling, normalization or scaling of the data is not required as it is done in ANN in the range [-1,1]. For the GP simulations, the population size and the number of generations are equal to the number of inputs as described in Table 1. For selection of parent genes from the pool of available solutions, a tournament selection strategy is adopted with size selected as 4 . The maximum depth of each tree is set to 5 to have more control over the evolved equations. The crossover, mutation and direct reproduction probabilities are taken as $0.85,0.1$ and 0.05 respectively.

Here a number of potential models are evolved at random and each model is trained and tested using the training and testing data respectively. The fitness of each model is determined by the RMSE between the predicted and actual value of output that is discharge $(\mathrm{Q})$ as the objective function given as:

$$
\mathrm{RMSE}=\sqrt{\frac{\sum_{\mathrm{i}=1}^{\mathrm{n}}\left(\mathrm{Q}_{\mathrm{m}}-\mathrm{Q}_{\mathrm{p}}\right)}{\mathrm{n}}}
$$

where, $n$ is the number of cases in the fitness group. If the errors calculated by using Eq. 2 for all models in the existing population do not satisfy the termination criteria, the generation of new population continues till the best model is developed as per the earlier discussion. In the GP model development it is important to make a tradeoff between accuracy and complexity in terms of the number of genes and depth of the GP tree. The developed GP model for all the five data sets are described as Eqs (3) to (7) as shown in Table 2.

\section{A. Prediction Using Data Set I}

In Fig. 5, first plot shows fitness, second shows prediction and third with bias -0.02085 as shown in Eq. 3) and gene values for smooth data set I. During several runs for smooth data the best fitness is achieved at generation 77 with minimum bias while RMSE for training as 0.020 and testing as 0.392 .
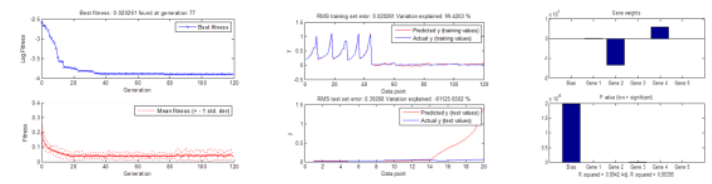

Figure 5. Plot for Fitness, Prediction along with Bias and Gene for Smooth data in GP

\section{B. Prediction Using Data Set II}

Fig. 6 shows fitness, prediction along with bias 0.1274

(as in Eq. 3) and gene values for rough data set II.
TABLE II. EQUATIONS DERIVED FOR DIFFERENT GP MODELS

\begin{tabular}{|c|c|c|}
\hline Model & Developed Equation & EqNo \\
\hline $\begin{array}{l}\text { Model I } \\
\text { (Smooth) }\end{array}$ & $\begin{aligned} Q= & 42.42 S_{0} R_{c} \alpha^{2}+\left(5.174 R_{+}^{2}+0.9396 R_{+}+0.7491 \beta-95.41 n_{c} \beta\right) \alpha \\
& -0.7491 \beta+0.9396 \tanh (\beta)-0.02085\end{aligned}$ & (3) \\
\hline $\begin{array}{l}\text { Model II } \\
\text { (Rough) }\end{array}$ & $\begin{aligned} Q= & 2.764 S_{0}-0.0129 \alpha-0.1594 \beta+2.923 R_{\tau}+0.07304 p \log (3.105) \\
& -3.357 \tanh \left(n_{f}\right)+0.1274\end{aligned}$ & (4) \\
\hline $\begin{array}{c}\text { Model III } \\
\text { (Smooth } \\
\text { and } \\
\text { Rough) }\end{array}$ & $\begin{aligned} Q= & 0.0008305 n_{c}-0.0008305 S_{0}-0.1078 \beta+0.6329 \alpha R_{q}+11.27 \alpha R_{f}^{2} \\
& -0.0008305 \alpha^{2}-206.2 \alpha n_{p} \beta R_{q}+0.001579\end{aligned}$ & (5) \\
\hline $\begin{array}{l}\text { Model IV } \\
\text { (River) }\end{array}$ & $\begin{aligned} Q= & 176.7 R_{\varphi}^{2} \tanh \left(R_{+}\right)+1610.0 \beta \tanh (\beta)-4.535 \times 10^{5} S_{0} \beta \\
& (p \operatorname{pog}(\beta)+\tanh (\alpha))+38.2 p \log \left(\alpha-R_{f}\right) \tanh (\beta)\left(\beta+R_{f}\right)-47.45\end{aligned}$ & (6) \\
\hline $\begin{array}{l}\text { Model V } \\
\text { (Smooth. } \\
\text { Rough } \\
\text { and } \\
\text { River) }\end{array}$ & $\begin{aligned} Q= & 590.6\left(n_{\varepsilon}+R_{q}\right)^{2}-1.137 \times 10^{6} n_{\varepsilon}^{2} R_{q}-18700.0 R_{q}^{2}\left(n_{q}+S_{0}\right) \\
& +2.294 \times 10^{6} n_{q} S_{0} R_{q}^{2}+6.517 \times 10^{5} n_{q}^{2} \beta R_{q}-3.052\end{aligned}$ & (7) \\
\hline
\end{tabular}

During the several runs for rough data, fitness is achieved with RMSE for training as 0.011 and for testing as 0.002 at generation 0 .
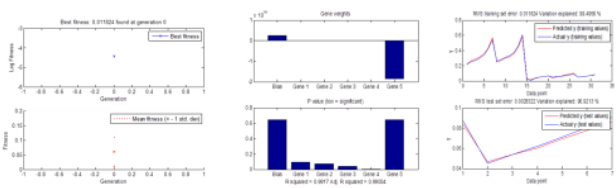

Figure 6. Plot for Fitness, Prediction along with Bias and Gene for Rough data set II in GP

\section{Prediction Using Data Set III}

Fitness, prediction curve including bias 0.01579 (shown in Eq. 4) and gene for data set III are shown in Fig. 7. Best fitness is achieved for this model at generation 12 with minimum bias along with RMSE training of 0.048 and testing of 0.130 .

\section{Prediction Using Data Set IV}

For River data best fitness is achieved at generation 48 with minimum bias -47.45 shown in Eq. (5) having RMSE for training of 71.718 and testing of 103.4 (Fig. 8).
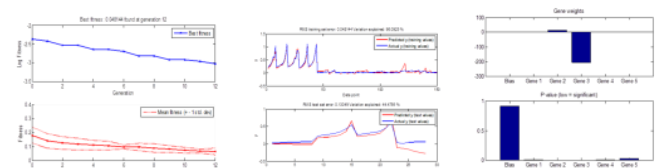

Figure 7. Plot for Fitness, Prediction along with Bias and Gene for Smooth and Rough data in GP
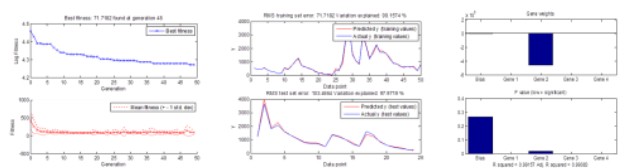

Figure 8. Fitness, Prediction Plot along with Bias and Gene for River data in GP

\section{E. Prediction Using Data Set V}

Similarly for data set V, (combination of all data) the best fitness is achieved at generation 206 with minimum bias $\quad-3.052$ (shown in Eq. 6) having RMSE for training as 21.628 and testing as 16.01 (Figure 9). 
Proc. of Eighth International Conference On Advances in Civil, Structural and Mechanical Engineering - ACSM 2018 Copyright $@$ Institute of Research Engineers and Doctors, USA. All rights reserved.

ISBN: 978-1-63248-154-2 doi: 10.15224/978-1-63248-154-2-10

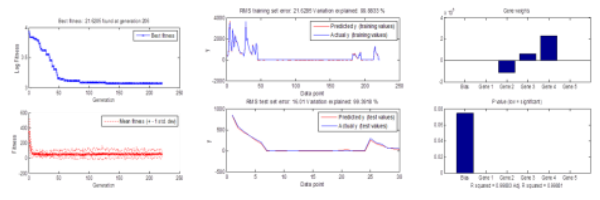

Figure 9. Plot for Fitness, Prediction along with Bias and Gene for data set $\mathrm{V}$ in GP

\section{F. Sensitivity Analysis}

This is an important aspect of a developed model to find out the relative effect of the input parameters. Different approaches have been suggested by researchers to select the important input variables. In the current study sensitivity analysis is made following Liong et al 2002 for all five data sets in GP. As per Liong, the sensitivity $\left(S_{\mathrm{i}}\right)$ of each parameter is determined by varying it while others are kept constant at the same time. It is expressed as,

$$
S_{i}=\frac{1}{N} \sum_{i}^{N}\left(\frac{\% \text { Change inoutput }}{\% \text { Change ininput }}\right) \times 100
$$

TABLE III. SENSITIVITY ANALYSIS AND RANKING

\begin{tabular}{|c|c|c|c|c|c|c|c|c|c|c|c|}
\hline \multirow[t]{2}{*}{ PARAMETER } & \multicolumn{2}{|c|}{ Data Set I } & \multicolumn{2}{|c|}{$\begin{array}{l}\text { Data } \\
\text { Set II }\end{array}$} & \multicolumn{2}{|c|}{$\begin{array}{l}\text { Data } \\
\text { Set III }\end{array}$} & \multicolumn{2}{|c|}{$\begin{array}{l}\text { Data } \\
\text { Set IV }\end{array}$} & \multicolumn{2}{|c|}{$\begin{array}{l}\text { Data } \\
\text { Set V }\end{array}$} & \multirow{2}{*}{$\begin{array}{l}\text { Rank } \\
\text { Index } \\
\Sigma(\mathbb{R})\end{array}$} \\
\hline & Value & $R_{1}$ & Value & $\mathrm{R}_{2}$ & Value & $\mathrm{R}_{3}$ & Value & $R_{4}$ & Value & $R_{5}$ & \\
\hline (1) & (2) & (3) & (4) & (5) & (6) & (7) & (8) & (9) & (10) & (11) & (12) \\
\hline$\alpha$ & 1.467 & 2 & -7.978 & 1 & 0.937 & 5 & 0 & 4 & 0 & 5 & 17 \\
\hline$N_{k}$ & 0 & 4 & -3.652 & 3 & -2.810 & 4 & -0.075 & 3 & 2.273 & 2 & 16 \\
\hline$S_{0}$ & 0 & 4 & -3.226 & 4 & -3.438 & 3 & 0 & 4 & 0.077 & 4 & 19 \\
\hline$\beta$ & 0.009 & 3 & -3.679 & 2 & -3.531 & 2 & 0.739 & 2 & -0.457 & 3 & 12 \\
\hline$R_{T}$ & 4.303 & 1 & -2.124 & 5 & 5.049 & 1 & 12.996 & 1 & 4.191 & 1 & 9 \\
\hline
\end{tabular}

All ranks corresponding to data sets are listed in Table 3 (columns 3, 5, 7, 9, and 11). Column 12 presents sensitivity analysis. As per GP, the hydraulics radius is the most important parameter found in case of all five models with least rank index of 9 followed by $\beta, n_{f p}, \alpha$ and $S_{o}$ in order of their ranking index.

\section{G. Comparison of Prediction by Soft Computing Models}

This section presents a comparison of prediction done by GP with other soft computing techniques described earlier. The Cascade neural network is not compared here as it has reported to have given very poor performance.

Performances of the models are judged by taking some global statistical parameters for the current analysis of all the models such as; Average Absolute Relative Error (AARE), Normalised Mean Bias Error (NMBE), Pearson's Correlation coefficient (R), Nash-Sutcliff efficiency (E) and Normalized Root Mean Square Error (NRMSE) (e.g. Srinivasulu et al. 2006). More details on these parameters are available in other reported works [7-9].

Performance parameters for GP, ANFIS, Fuzzy and CES are calculated and given in Table 4 for comparison. The results from the soft computing models namely ANFIS and GP are compared. As Fuzzy and CES give higher percentage of errors in their predictions in comparison to ANFIS and GP, they are excluded from the comparison plots for clarity reasons.
TABLE IV. EVAluation of Performance Parameters AND RANKING FOR ALL MODELS

\begin{tabular}{|c|c|c|c|c|c|c|c|c|c|c|c|c|}
\hline Data Se & Methods & AARE & $R_{1}$ & NMBE & $R_{2}$ & $\mathrm{R}$ & $R_{3}$ & E & $\mathbf{R}_{\text {, }}$ & NRMSE & $\mathrm{R}_{\mathrm{s}}$ & $\begin{array}{l}\text { RankIndex } \\
\text { (Sum } R_{1}: R\end{array}$ \\
\hline Datá & & (1) & (2 & (3) & (4) & (5) & (6) & (7) & (8) & (9) & (10) & (11) \\
\hline \multirow{4}{*}{ I } & GP & 21.041 & 2 & -0.158 & 2 & 0.838 & 3 & 0.999 & 1 & 0.288 & 1 & 9 \\
\hline & ANFIS & 22.876 & 3 & 0.159 & 3 & 0.934 & 1 & 0.526 & 3 & 0.298 & 2 & 12 \\
\hline & CES & 18.116 & 1 & -0.068 & 1 & 0.895 & 2 & 0.836 & 2 & 0.506 & 3 & 9 \\
\hline & FUZZI & 57.744 & 4 & 1.677 & 4 & 0.113 & 4 & -0.955 & 4 & 2.028 & 4 & 20 \\
\hline \multirow{4}{*}{ II } & GP & \begin{tabular}{|l}
5.727 \\
\end{tabular} & 1 & \begin{tabular}{|c|}
-0.018 \\
\end{tabular} & 2 & 0.205 & 4 & 1.000 & 1 & 0.005 & 1 & 9 \\
\hline & ANFIS & 20.932 & 3 & \begin{tabular}{|l|}
0.013 \\
\end{tabular} & 1 & 0.465 & 1 & 0.968 & 2 & 0.007 & 2 & 9 \\
\hline & CES & \begin{tabular}{|l|l|}
14.993 \\
\end{tabular} & 2 & \begin{tabular}{|l|l|} 
\\
\end{tabular} & 3 & 0.464 & 2 & 0.345 & 3 & 0.936 & 3 & 13 \\
\hline & FUZZI & 45.745 & 4 & 2.724 & 4 & 0.314 & 3 & -221.0 & 4 & 3.312 & 4 & 19 \\
\hline \multirow{4}{*}{ III } & GP & 37.225 & 3 & 0.040 & 1 & 0.991 & 1 & 0.980 & 1 & 0.169 & 1 & 7 \\
\hline & ANFIS & 26.874 & 2 & \begin{tabular}{|l|}
-0.162 \\
\end{tabular} & 2 & 0.972 & 3 & 0.954 & 2 & 0.255 & 2 & 11 \\
\hline & CES & 18.001 & 1 & \begin{tabular}{|l|}
0.168 \\
\end{tabular} & 3 & 0.992 & 2 & 0.920 & 3 & 0.336 & 3 & 12 \\
\hline & FUZZI & 132.73 & 4 & \begin{tabular}{|l|l} 
\\
\end{tabular} & 4 & 0.868 & 4 & 0.791 & 4 & 0.885 & 4 & 20 \\
\hline \multirow{4}{*}{ IV } & GP & 3.016 & 1 & \begin{tabular}{|l|}
-0.013 \\
\end{tabular} & 2 & 0.996 & 2 & 0.991 & 2 & 0.047 & 2 & 9 \\
\hline & ANFIS & 3.346 & 2 & \begin{tabular}{|l|}
0.011 \\
\end{tabular} & 1 & 0.997 & 1 & 1.000 & 1 & 0.046 & 1 & 6 \\
\hline & CES & \begin{tabular}{|l|l|}
142.63 \\
\end{tabular} & 4 & \begin{tabular}{|l|l|} 
\\
\end{tabular} & 4 & 0.994 & 3 & -0.827 & 4 & 0.664 & 3 & 18 \\
\hline & FUZZI & 7.014 & 3 & 0.023 & 3 & 0.986 & 4 & 0.968 & 3 & 0.088 & 4 & 17 \\
\hline \multirow{4}{*}{ V } & GP & 75.926 & 3 & 0.188 & 3 & 0.592 & 4 & 0.997 & 1 & 0.146 & 1 & 12 \\
\hline & ANFIS & 84.835 & 4 & \begin{tabular}{|c|}
-0.061 \\
\end{tabular} & 2 & 0.877 & 1 & 0.992 & 2 & 0.352 & 3 & 12 \\
\hline & CES & 42.954 & 1 & -0.512 & 4 & 0.872 & 3 & 0.660 & 4 & 1.067 & 4 & 16 \\
\hline & FUZZ) & 74.026 & 2 & -0.002 & 1 & 0.874 & 2 & 0.991 & 3 & 0.175 & 2 & 10 \\
\hline
\end{tabular}

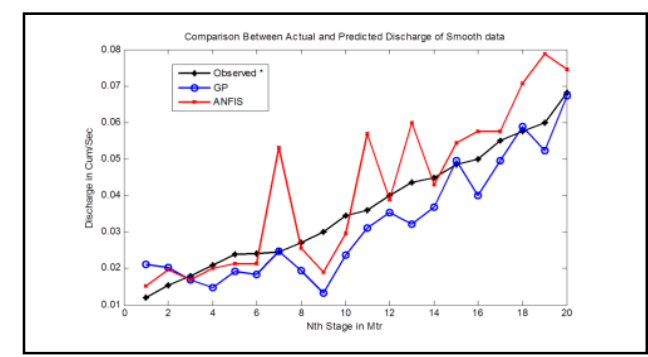

Figure 10. Predicted discharge using GP and ANFIS for Data Set I

The Fig. 11 shows the prediction of discharge by GP and ANFIS along with observed discharge for data set I. ANFIS shows little over prediction while GP makes slightly under prediction. However for all stage values, GP has shown better prediction following the trend of observed data. The ranking of evaluation parameter performance of AARE (column 2), NMBE (column 4), R (column 6), E in column 8 and NRMSE ( column 10) are given in Table 5 . It can be safely concluded that, whether in individual ranking or in group rank index, GP and CES scores the highest rank (column 11 of Table 5).

Fig. 12 shows the prediction using rough data set II. Prediction by GP is very close to the observed value than ANFIS for all stages. As GP bears lowest AARE (5.7), NMBE (-0.018), $E$ value equal to 1.0 , hence it's performance is found to be the best. NRMSE for GP is better (0.005) when compared to ANFIS. The Fig. 13 depicts the plot for smooth and rough data. From Table 5, it is also quite clear that GP and ANFIS give better modelled rank performance than CES and FUZZY.

The Fig. 14 presents prediction plot for data of Brahmani river. As per rank index, GP performs poor than ANFIS but is better than CES and FUZZY. The prediction graphs of Fig. 15 is for the combined data which contains very low as well as very high discharge values of stage in increasing order. Up to 30 test data points its very low as shown in the Figure 15. Beyond 32 to 50, it represents very high magnitude of flow. Rank value got for GP and ANFIS are same but, from the plots it is clear that GP follows the 
Proc. of Eighth International Conference On Advances in Civil, Structural and Mechanical Engineering - ACSM 2018 Copyright (@) Institute of Research Engineers and Doctors, USA. All rights reserved.

ISBN: 978-1-63248-154-2 doi: 10.15224/978-1-63248-154-2-10

same trend as that of observed discharge whereas the ANFIS model exhibits bit over prediction.

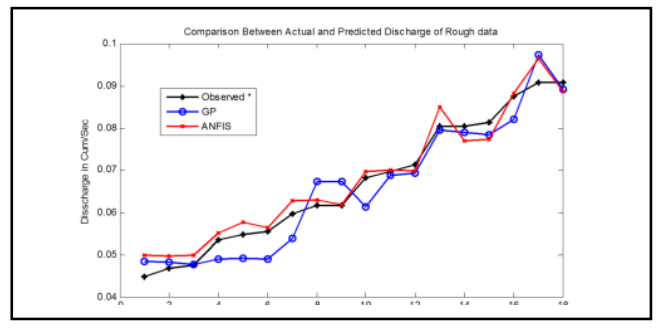

Figure 11. Predicted discharge using GP and ANFIS for Data Set II

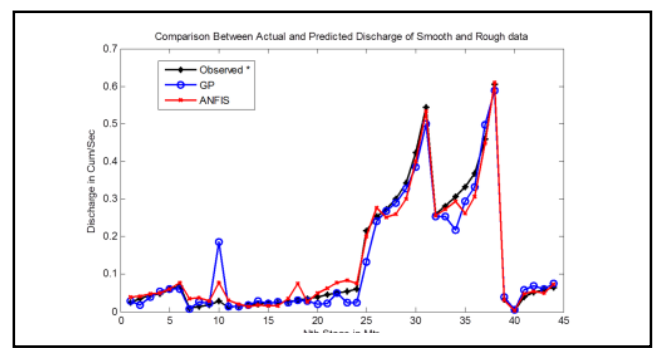

Figure 12. Predicted discharge using GP and ANFIS for Data Set III

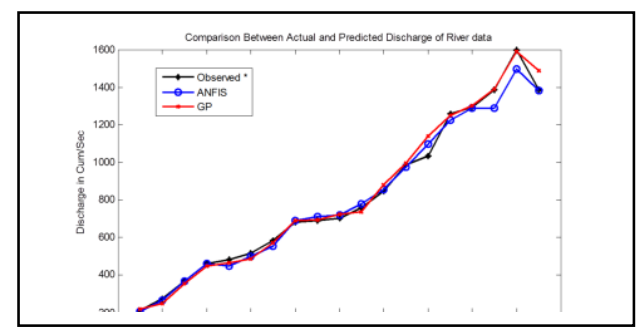

Figure 13. Predicted discharge using GP and ANFIS of Data Set IV

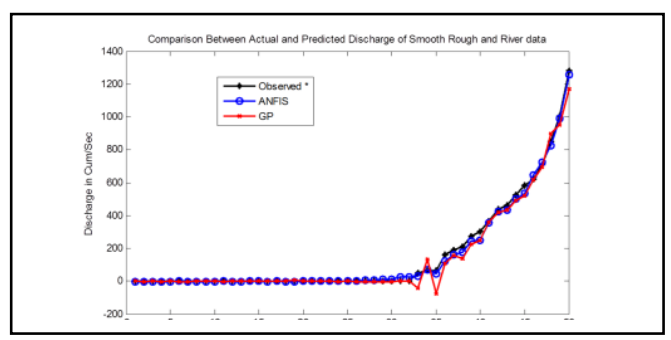

Figure 14. Predicted discharge using GP and Data Set V

The overall performance of models is ranked and final rank index is calculated for each data set with respect to all the ranking criteria. Least value of rank index justifies the goodness of the model. GP rank index varies from 7 to 12 where as ANFIS rank index values vary from 6 to 12 as shown in Table 5. The rank index for CES from 9 to 18 and the same for FUZZY varies from 10 to 20. From final rank index in Table 5 (column 7) it is inferred that GP model exhibits consistent performance for all the five data sets while others show variable performance. Thus, it can be concluded that the Genetic Programming can perform well in predicting a wide range of flow in the river system.

TABLE V. Final RanKING OF All Models

\begin{tabular}{|c|c|c|c|c|c|c|}
\hline Models & Data Set I & Data Set II & Data Set III & Data Set IV & Data Set V & Rank Inder \\
\hline (1) & $(2)$ & $(3)$ & $(4)$ & $(5)$ & $(6)$ & $(7)$ \\
\hline GP & 9 & 9 & 7 & 9 & 12 & 46 \\
\hline ANFIS & 12 & 9 & 11 & 6 & 12 & 50 \\
\hline CES & 9 & 13 & 12 & 18 & 16 & 68 \\
\hline FUZZY & 20 & 19 & 20 & 17 & 10 & 86 \\
\hline
\end{tabular}

\section{Acknowledgment}

The writers acknowledge the Central Water Commission, Bhubaneswar for providing river Brahmani data for the analysis. The paper is part of the research work carried out in the National Institute of Technology, Rourkela.

\section{References}

[1] K. K.Khatua, K. C. Patra and P. K. Mohanty; "Stage Discharge Prediction for Straight and Smooth Compound Channels with Wide Floodplains", J. Hydraul. Eng., ASCE, vol. 138(1): 93-99 (2012).

[2] K. C. Patra,. "Flow Interaction of Meandering river with Flood Plains", $P h D$ Thesis, IIT, KGP (1999).

[3] A. K. Kordon. Future Trends in Soft Computing Industrial Applications. Proceedings of the 2006 IEEE Congress on Evolutionary Computation. 7854-7861 (2006)

[4] A.J. Abebe and R.K.Price. Information Theory and Neural Networks for Managing Model Uncertainty in Flood Routing. Journal of Computing in Civil Engineering, ASCE, 18(4): 377-380 (2004).

[5] M. T.Dastorani, A. Moghadamnia J.Piri and Rico- Ramirez; "Miguel. Application of ANN and ANFIS Models for Reconstructing Missing Flow Data", Environ Monit Assess, Vol. 166: 421-434(2010).

[6] R.R Shrestha, S.Theoblad, and F.Nestmann, "Simulation of Flood Flow in a River System using Artificial Neural Networks ", Hydrology and Earth System Sciences, Vol.9, no.4, 313-321 (2005).

[7] Alok Adhikari, K. C. Patra and S. K. Das. Prediction of Discharge with Elman and Cascade Neural Networks. Reacearch Journal of Recent Sciences, Vol.2 (ISC-2012):279-284 (ISSN 2277-2502) (2013).

[8] A. Adhikari, K.C.Patra and K. K. Khatua Stage Discharge Prediction using RBF and Elman Neural Networks. Advances in Engineering and Technology Series, (IEEE ICARET).Vol. 2: 1-6 (2013).

[9] A. Adhikari, K. C. Patra and N.Adhikari. Prediction of Discharge in Straight Compound Channels using Conventional and Soft Computing Tools. Journal of Engg Research and Application, Vol.7 (8): 42-51(2017).

[10] D.W.Knight, School of Civil Engineering ,The University of BirminghamConveyance in 1D River Models, A report for HR Wallingford \& The Environment Agency (2001).

[11] W.R.C.Myers, "Velocity and Discharge in Compound Channels", Journal of Hydraulic Engineering, ASCE, Vol. 113(6), pp. 753-766 (1987).

[12] S. A. Atabay and D. W. Knight., " The influence of floodplain width on the stage-discharge relationship for compound channels", River Flow 2002, Proc. Int. Conf. on Fluvial Hydraulics, Louvain-la-Neuve, Belgium, Vol. 1: 197-204 (2002).

[13] B.Rezaei, "Overbank flow in compound channels with prismatic and nonprismatic floodplains", Diss. University of Birmingham, (2006).

[14] J R. Koja.; "Genetic Programming on the programming of the computers by means of natural selection", The MIT Press, USA (1992).

[15] Md.H. Azamathulla and Ab Ghani Aminuddin; "Genetic Programming to Predict River Pipeline", Scour. Journal of Pipeline Systems Engineering and Practice, ACSE, Vol.1, No. 3: 127-132 (2010).

[16] D. P.Searson, David E. Leahy and Mark J. Willis. "GPTIPS: An Open Source Genetic Programming Toolbox for Multigene Symbolic Regression"; Proc. Of Int. Conference of Engineering and Computer Scientists, Vol. I, IMECS2010, March17-19 (2010).

[17] J.Shing, and Roger Jang. "ANFIS: Adaptive- Network-Based FUZZY Inference System”, IEE Transaction on Systems, MAN, and Cybernetics, Vol. 23, No 3, May/ June, 665-685 (1993).

[18] M. Erol Keskin, Dilek Taylan and O.Terzi; "Adaptive Neural-based Fuzzy Inference System (ANFIS) approach for modelling Hydrological Time Series”, Hydrological Sciences Journal, Vol. 51(4): 588-988 (2010).

\footnotetext{
About Author (s):
}

Alok Adhikari: A research scholar in Departement of Water Resources Engineering, National Institute of Technology, Rourkela, India. He has done M.Tech in Soil and Water Conservation Engineering from OUAT, Bhubaneswar, India.

Dr Nibedita Adhikari is attached to Biju Patnaik University of Technology, Odisha, India. Her research area includes Soft Computing and Paralle computing.

Dr K. C. Patra is working as Professor in Department of Civil Engineering, NIT Rourkela, India. 